\title{
MộT SỐ PHƯƠNG PHÁP NÂNG CAO Độ PHÂN GIẢI ẢNH VIỄN THÁM BẰNG PHƯƠNG PHÁP SIÊU PHÂN GIẢI
}

\author{
TS. LÊ QUÓC HƯNG, KS. TRƯƠNG TH! TUYÉT \\ Cục Viễn thám quốc gia
}

\section{Tóm tắt:}

Nâng cao độ phân giải không gian của ảnh luôn là một trong những mục tiêu nghiên cứu hàng đầu của các nhà khoa học trong lĩnh vực viễn thám. Biện pháp nâng cao độ phân giải không gian bằng phương pháp nâng cấp, nghiên cứu chế tạo các đầu thu, bộ cảm mới tiên tiến cho phép thu ảnh với độ phân giải không gian cao có chí phí vô cùng lớn. Bên cạnh đó, khi nâng cao độ phân giải không gian thì độ phân giải phổ thường bị hạn chế. Những nguyên do trên đã thúc đẩy các nhà khoa học nghiên cứu phương pháp xử lý nâng cao độ phân giải không gian bằng các thuật toán chuyển đổi ảnh từ độ phân giải thấp về độ phân giải cao dựa trên chính các dữ liệu ảnh thu thập được. Bài báo tập trung đánh giá một số phương pháp siêu phân giải (Superresolution, viết tắt $S R$ ) phát triển còn khá mới mẻ trong thời gian gần đây cho mục đích nâng cao độ phân giải nhằm tận dụng tốt hơn thông tin ảnh viễn thám VNREDSat-1 của Việt Nam.

\section{1. Đặt vấn đề}

Hệ thống vệ tinh VNREDSat-1 là hệ thống vệ tinh quan sát Trái Đất đầu tiên của Việt Nam. Sau khi thực hiện thành công Dự án, Việt Nam hiện nay và sau này sẽ chủ động cung cấp ảnh viễn thám độ phân giải cao cho các Bộ, ngành và các tỉnh thành có nhu cầu sử dụng dữ liệu ảnh viễn thám phục vụ phát triển kinh tế - xã hội, ứng phó với thảm hoạ thiên nhiên và biến đổi khí hậu. Để tận dụng và ứng dụng có hiệu quả nguồn ảnh viễn thám đầu tiên của Việt Nam cho mục đích giám sát tài nguyên thiên nhiên và môi trường, bảo vệ lãnh thổ, việc nghiên cứu nâng cao độ phân giải không gian là cần thiết. Việc nâng cao độ phân giải không gian theo hướng xử lý một chuỗi các ảnh trên chụp cùng một vị trí, có cùng độ phân giải để tạo ra một ảnh có độ phân giải cao hơn. Phương pháp này được gọi là phương pháp siêu phân giải - supperresolution (SR)

Kỹ thuật quan trọng nhất trong phương pháp SR là phương pháp nội suy làm tăng hay giảm kích thước pixel, sử dụng đa ảnh độ phân giải thấp để tái cấu trúc, xử lý các hạn chế của ảnh đơn tốt hơn. Các thông tin dư thừa chứa trong các ảnh độ phân giải thấp có thể được đưa vào bằng sự tịnh tiến giữa các subpixel giữa chúng. Sự tịnh tiến của subpixel này có thể xuất hiện theo các đối tượng hoặc theo sự chuyển động được điều khiển ví dụ như hệ thống thu ảnh trên vệ tinh với quỹ đạo có tốc độ và phương được ấn định trước.

\section{Các phương pháp siêu phân giải}

Hệ thống chụp ảnh luôn tồn tại các sai số do hạn chế về phần cứng, ảnh thu nhận được luôn bị suy giảm chất lượng. Ví dụ, giới hạn về độ mở của ống kính chính là nguyên nhân dẫn tới hiện tượng mờ ảnh, hiện tượng này thường được mô hình hóa bởi hàm lan truyền theo điểm nguồn PSF (Point Spread Function). Hạn chế thời gian phơi sáng thể hiện rất rõ khi chụp những đối tượng chuyển động hoặc thiết bị chụp chuyển động. Hạn chế về kích 
thước bộ cảm sẽ đưa đến ảnh hưởng mờ do bộ cảm. Ảnh hưởng này xuất phát từ việc giá trị pixel ảnh chỉ được dựa vào một xung trên một đơn vị diện tích mà bộ cảm thu nhận thay vì lấy mẫu xung từ nhiều bộ cảm. Hạn chế về mật độ cảm biến trên bộ cảm sẽ dẫn tới hiện tượng răng cưa và từ đó làm ảnh hưởng tới độ phân giải không gian của tấm ảnh thu nhận được. Những ảnh hưởng trên được mô phỏng hoàn toàn hoặc một phần trong các phương pháp SR khác nhau. Kỹ thuật siêu phân giải (SR) là phương pháp xây dựng ảnh độ phân giải cao $(\mathrm{HR})$ từ một vài ảnh độ phân giải thấp (LR) do đó tăng cường các thành phần có tần số cao và loại bỏ các yếu tố gây suy giảm chất lượng ảnh bằng cách xử lý các ảnh từ các bộ cảm có độ phân giải thấp.

\subsection{Mô hình chụp ảnh}

Hình dưới mô tả một hệ thống thu ảnh được mô tả theo [2] với đầu vào là những ảnh tự nhiên liên tiếp với chất lượng đạt tới giới hạn của hệ thống.

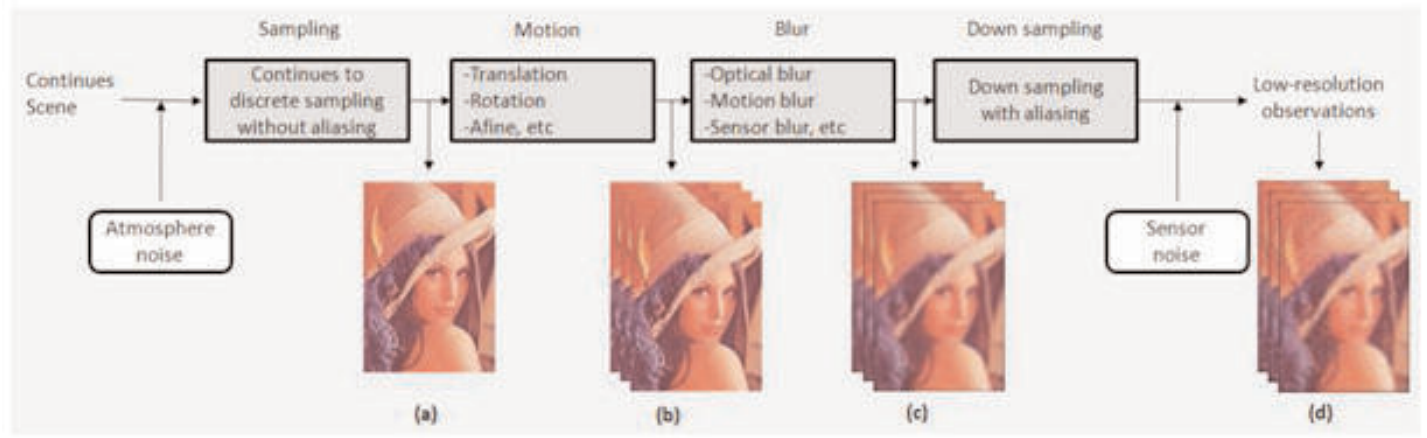

Hình 1: Hệ thống tạo ảnh từ đầu thu

Nếu xem $X$ là ảnh độ phân giải cao kỳ vọng và $Y_{k}$ là ảnh thứ $k$ độ phân giải thấp từ hệ thống chụp ảnh. Khi đó, mối quan hệ giữa $X$ và $Y_{k}$ được mô tả theo một hàm "lexicographical". Giả sử, ta thu được $K$ ảnh LR của $X$, ở đó ảnh độ phân giải thấp quan hệ với ảnh độ phân giải cao theo công thức:

$$
Y_{k}=D_{k} H_{k} F_{k} X+V_{k}, k=1,2,3 \ldots K
$$

Trong đó: $F_{k}$ là hàm mô tả chuyển động của ảnh thứ $k$

$\mathrm{H}_{\mathrm{k}}$ là mô hình ảnh hưởng của hiện tượng mờ ảnh.

$D_{k}$ là toán tử down-sampling (toán tử làm mịn ảnh).

$V_{\mathrm{k}}$ là hàm nhiễu.

Hàm quan hệ tuyến tính trên có thể được thể hiện theo công thức dưới dạng ma trận:

$$
\left[\begin{array}{c}
Y_{1} \\
Y_{2} \\
\cdot \\
\dot{Y_{K}}
\end{array}\right]=\left[\begin{array}{ccc}
D_{1} & H_{1} & F_{1} \\
D_{2} & H_{2} & F_{2} \\
: & : & : \\
\dot{D_{K}} & \dot{H}_{K} & \dot{F}_{K}
\end{array}\right] X+\left[\begin{array}{c}
V_{1} \\
V_{2} \\
\cdot \\
\dot{V_{K}}
\end{array}\right]
$$

Hay $\mathbf{Y}=\mathbf{M} \cdot \mathbf{X}+\underline{\mathbf{V}}$ 
Trong thực tế, ma trận $\mathrm{M}$ thường là ma trận thưa nên hàm liên kết thường rất yếu. Hơnnữa, các thông số $\mathrm{D}, \mathrm{H}, \mathrm{F}$ thường không có đầy đủ mà phải tính toán dựa trên các số liệu thực tế. Do đó, việc tính toán ảnh tạo độ phân giải cao $X$ là không hề đơn giản. Các nhà khoa học đã và đang cố gắng tìm các phương pháp tổ hợp ảnh độ phân giải thấp $Y$ theo nhiều phương pháp toán học nhằm tìm hàm quan hệ thực tế khả dụng hơn.

\subsection{Phương pháp siêu phân giải sử dụng miền tần số}

Tsai và Huang [1] đã sử dụng mối quan hệ của ảnh độ phân giải cao với các ảnh độ phân giải thấp được tịnh tiến bởi công thức miền tần số dựa trên sự dịch chuyển tịnh tiến của các ảnh độ phân giải thấp và các thuộc tính khử răng cưa của chuỗi biến đổi Fourier.

Nếu $x\left(\mathrm{t}_{1}, \mathrm{t}_{2}\right)$ biểu thị cho một cảnh độ phân giải cao trong chuỗi liên tục thì sẽ có một trường $\mathrm{K}$ ảnh tịnh tiến:

$$
\mathrm{x}_{\mathrm{k}}\left(\mathrm{t}_{1}, \mathrm{t}_{2}\right)=\mathrm{x}\left(\mathrm{t}_{1}+\Delta \mathrm{k}_{1}, \mathrm{t}_{2}+\Delta \mathrm{k}_{2}\right), \text { với } \mathrm{k}=1,2, \ldots, \mathrm{K}
$$

Trong đó: $\Delta \mathrm{k}_{1}$ và $\Delta \mathrm{k}_{2}$ là tùy biến theo mức độ dịch chuyển.

Chuỗi liên tục biến đổi Fourier (CFT) của một cảnh được cho bởi $X\left(\mathrm{u}_{1}, \mathrm{u}_{2}\right)$ và những ảnh tính chuyển từ nó là $X_{k}\left(\mathrm{u}_{1}, \mathrm{u}_{2}\right)$. Mối quan hệ của chúng sẽ được xác định theo công thức:

$$
X_{k}\left(\mathrm{u}_{1}, \mathrm{u}_{2}\right)=\exp \left[j 2 \pi\left(\Delta \mathrm{k}_{1} \mathrm{u}_{1}+\Delta \mathrm{k}_{2} \mathrm{u}_{2}\right)\right] X\left(\mathrm{u}_{1}, \mathrm{u}_{2}\right)
$$

Ảnh tịnh tiến được lấy mẫu theo dạng xung trong thời gian $T 1$ và T2 để có được các ảnh độ phân giải thấp:

$$
y_{k}\left[n_{1}, n_{2}\right]=x\left(n_{1} \cdot T_{1}+\Delta k_{1}, n_{2} \cdot T_{2}+\Delta k_{2}\right),
$$

với $\mathrm{n}_{1}=0,1,2, \ldots, \mathrm{N}_{1}-1 ; \quad \mathrm{n}_{2}=0,1,2, \ldots, \mathrm{N}_{2}-1$.

Xét hàm Chuỗi rời rạc của các biến đổi Fourier (DFTs) của các ảnh độ phân giải thấp $Y$ $\mathrm{k}[\mathrm{r} 1, \mathrm{r} 2]$. Biến đổi $\mathrm{CFT}$ của các ảnh tịnh tiến có mối quan hệ với biến đổi DFTs thông qua các thuộc tính khử răng cưa của chuỗi biến đổi Fourier:

$$
Y_{k}\left[r_{1}, r_{2}\right]=\frac{1}{T_{1} T_{2}} \sum_{m_{1}=-\infty}^{\infty} \sum_{m_{2}=-\infty}^{\infty} X_{k}\left(\frac{2 \pi}{T_{1}}\left(\frac{r_{1}}{N_{1}}-m_{1}\right), \frac{2 \pi}{T_{2}}\left(\frac{r_{2}}{N_{2}}-m_{2}\right)\right)
$$

Giả định $X\left(\mathrm{u}_{1}, \mathrm{u}_{2}\right)$ là giới hạn, $\left|X\left(\mathrm{u}_{1}, \mathrm{u}_{2}\right)\right|=0$ đối với $\left|\mathrm{u}_{1}\right| \geq\left(\mathrm{N}_{1} \pi\right) / \mathrm{T}_{1},\left|\mathrm{u}_{2}\right| \geq\left(\mathrm{N}_{2} \pi\right) / \mathrm{T}_{2}$, tổ hợp 02 công thức trên ta sẽ có hệ số DFT của $Y_{k}\left[r_{1}, r_{2}\right]$ với giá trị mẫu chưa biết của CFT $\mathrm{x}\left(\mathrm{t}_{1}, \mathrm{t}_{2}\right)$ theo dạng:

$$
Y=\Phi \cdot X
$$

Trong đó: $Y$ là vector $K$ hàng 1 cột với $k^{\text {th }}$ là hệ số của hàm DFT $Y_{k}\left[r_{1}, r_{2}\right]$

$X$ là vector $\mathrm{N}_{1} \mathrm{~N}_{2}$ hàng 1 cột với các giá trị mẫu chưa biết của hệ số hàm CFT $x\left(\mathrm{t}_{1}, \mathrm{t}_{2}\right)$

$\Phi$ là ma trận $\mathrm{K}$ hàng $\mathrm{N}_{1} \mathrm{~N}_{2}$ cột, là ma trận quan hệ giữa $Y$ và $X$

Công thức (*) định nghĩa quan hệ tuyến tính xuất phát từ việc hướng tới $X$ và sau đó 
sử dụng nghịch đảo hàm DFT để tái cấu trúc ảnh. Mối quan hệ trên sử dụng để tái cấu trúc ảnh SR với giả định là không có nhiễu và các dịch chuyển đã biết thông số đầy đủ. Quá trình giảm độ phân giải được giả định là sử dụng các mẫu xung có ảnh hưởng của hiện tượng mờ do bộ cảm viễn thám.

Phương pháp miền tần số bị giới hạn đối với sự tịnh tiến giữa các ảnh và sự thiếu rõ nét bất biến (spatially-invariant blurring). Vì thế, phương pháp này không thể sử dụng được để kết hợp các ảnh chụp ở các góc khác nhau do các ảnh có thể bị quay theo ảnh khác gây ra biến dạng và có khoảng cách lấy mẫu bất qui tắc.

\subsection{Phương pháp siêu phân giải miền không gian}

Hiện tồn tại rất nhiều phương pháp thống kê linh hoạt được ứng dụng [3], [4] để tái cấu trúc ảnh độ phân giải thấp. Công thức quan hệ giữa ảnh độ phân giải cao và ảnh độ phân giải thấp khá giống với phương pháp phục hồi ảnh truyền thống [5], rất nhiều phương pháp thống kê linh hoạt được ứng dụng để tái cấu trúc ảnh độ phân giải thấp. Có thể kể đến các phương pháp như Maximum Likelihood (ML), Maximum a Posteriori (MAP) [6] và phương pháp Projection Onto Convex Sets (POCS) [7] .

\subsubsection{Nội suy phục hồi ảnh - Phương pháp không tính lặp}

Giả định $\mathrm{H}_{\mathrm{k}}$ là không gian tuyến tính bất biến - Linearly Spatial Invariant (LSI) và đồng nhất đối với mọi khung hình $\mathrm{K}$, biểu thị không gian $\mathrm{H}$. Giả sử $\mathrm{F}_{\mathrm{k}}$ chỉ là mô hình chuyển động đơn giản như tịnh tiến, quay, khi đó $H$ và $F_{k}$ theo [8] thì:

$$
Y_{k}=D_{k} F_{k} H X+V_{k}=D_{k} F Z, \quad k=1,2, \ldots, K,
$$

Đây là cơ sở của phương pháp tính không lặp dựa trên nội suy và phục hồi ảnh. Có 3 bước cụ thể như sau:

- Đăng ký ảnh độ phân giải thấp (khớp các ảnh độ phân giải thấp).

- Nội suy không đồng dạng để xác định Z.

- Khử mờ và nhiễu để có $X$

Khung ảnh độ phân giải thấp được liên kết đầu tiên bởi một vài nguyên lý phục hồi ảnh để đưa ra các subpixel chính xác. Sự liên kết của các khung hình độ phân giải thấp sau đó được đặt trong một lưới độ phân giải cao mà ở đó nội suy không đồng dạng được dùng để lấp các pixel bị mất trên ảnh độ phân giải cao để có được $Z$. Sau cùng, $Z$ được tiến hành xóa mờ bởi phương pháp deconvonlutional truyền thống với việc loại bỏ nhiễu để tạo ra X.

Kenren, Peleg, and Brada [9] giới thiệu phương pháp tái cấu trúc ảnh SR thông qua 2 bước dựa trên mô hình chuyển động xoắn. Bên cạnh đó, Gross [10] đã đưa ra phương pháp nội suy không đồng dạng để tổ hợp bộ ảnh độ phân giải thấp bằng cách thiết lập nguyên lý tái chia mẫu đa kênh. Còn Papulis tiến hành phương pháp tái chia mẫu sử dụng hiệu ứng xóa mờ. (Xem hình 2)

Phương pháp nội suy cũng được liên tục cải tiến, có thể sử dụng phương pháp nội suy tam giác bất quy tắc, nội suy láng giềng gần nhất. Ưu điểm của phương pháp này là quá trình đăng ký ảnh xử lý dễ dàng tuy nhiên vẫn còn hạn chế trong việc giảm hiện tượng răng cưa trên ảnh. 


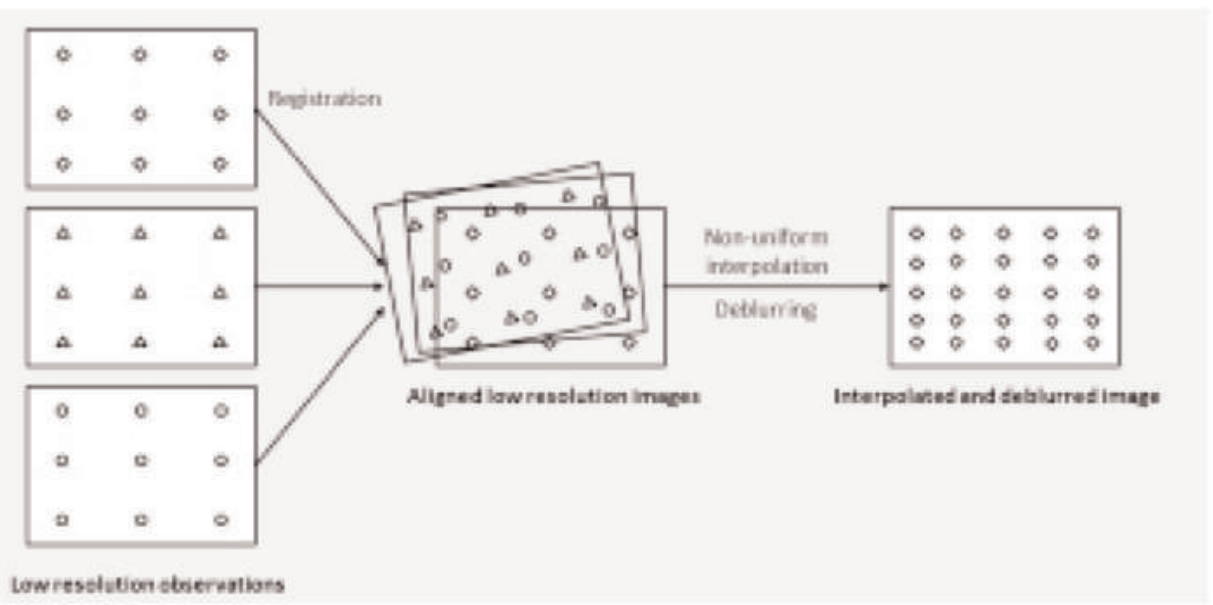

Hình 2: Phương pháp miền không gian nội suy - phục hồi ảnh

\subsubsection{Phương pháp thống kê}

Không như phương pháp nội suy - phục hồi ảnh, phương pháp thống kê xem các bước tái cấu ảnh $S R$ là ngẫu nhiên. Ảnh độ phân giải cao kết quả (HR) và các yếu tố đầu vào của sự dịch chuyển giữa các ảnh thu nhận được $(\mathrm{SR})$ có thể được xem như là các biến ngẫu nhiên. Đặt $M(v, h)$ là ma trận suy giảm được xác định bởi vector chuyển động $v$ và toán tử mờ $h$, tái cấu trúc ảnh SR có thể được đưa vào dạng của hàm Bayesian đầy đủ:

$$
\begin{gathered}
X=\arg \max _{X} \operatorname{Pr}(X \mid \underline{Y}) \\
=\arg \max _{X} \int_{v, h} \operatorname{Pr}(\underline{Y} \mid X, M(v, h)) \operatorname{Pr}(X) \operatorname{Pr}(M(v, h) d v
\end{gathered}
$$

Chú ý rằng, $X$ và $M(v, h)$ và được thống kê độc lập theo [11]. Ở đây, $\operatorname{Pr}(X, M(v, h))$ là dữ liệu thông tin ảnh, $\operatorname{Pr}(X)$ là hạn sai ám chỉ ảnh độ phân giải cao mong muốn, $\operatorname{Pr}(M(v, h))$ là thuật ngữ thể hiện sự thống kê của chuyển động. $\underline{V}$ trong công thức trên thường là chỉ độ nhiễu, giả định là bằng không và với một vector Gaussian rỗng. Theo đó:

$$
\operatorname{Pr}(\underline{Y} \mid X, M(v, h)) \propto \exp \left\{-\frac{1}{2 \sigma^{2}}\|\underline{Y}-M(v, h) X\|^{2}\right\}
$$

$\operatorname{Pr}(X)$ được xác định bằng cách sử dụng hàm phân phối Gibbs theo hàm mũ dạng:

$$
\operatorname{Pr}(X)=\frac{1}{Z} \exp \{-\alpha A(X)\}
$$

Trong đó: $A(X)$ là một hàm khả năng không âm; $Z$ là yếu tố chuẩn hóa.

Hàm Bayesian theo công thức ${ }^{*}$ ) rất phức tạp và rất khó để có thể thực hiện đánh giá thống kê khi vệ tinh chuyển động.

\subsubsection{Phương pháp thiết lập nguyên lý phục hồi ảnh}


Bên cạnh các phương pháp tiếp cận tối ưu hóa từ việc sử dụng biến ngẫu nhiên như đã thảo luận ở trên, một chiều hướng tiếp cận khác thông qua Phép chiếu trên tập lồi Projection onto Convex Sets (POCS) ) được giới thiệu trong [12]. Phương pháp POCS giải quyết vấn đề của ảnh độ phân giải thấp bằng cách xây dựng một tập lồi với nhiều giới hạn trong đó bao gồm cả yêu cầu về ảnh, yêu cầu này được xem như một điểm lồi. Những định nghĩa về tập lồi tương đối linh hoạt và có thể kết hợp nhiều loại điều kiện hoặc đặt trước, thậm chí là những giới hạn phi tuyến và không có tham số.

Tích hợp dữ liệu với những điều kiện tái cấu trúc có thể được mô hình hóa dưới dạng tập lồi K:

$$
C k=\left\{\boldsymbol{X} \mid\left\|D_{k} H_{k} F_{k} \boldsymbol{X}-\mathbf{Y}_{\mathrm{k}}\right\|^{2} \leq \sigma^{2}, 1 \leq k \leq K\right\}
$$

Trong đó, tồn tại điều kiện làm mịn và điều kiện biên độ. Kỹ thuật POCS đưa ra thuật toán tìm các điểm giao của tập $X$ :

$$
X_{k}+1=P_{M} P_{M-1} \ldots . P_{2} P_{1} X_{k}
$$

Trong đó, $\mathrm{X}_{0}$ là giá trị đặt ban đầu; $\mathrm{P}_{\mathrm{i}}$ là toán tử chiếu - toán tử chiếu này chiếu một điểm lên một tập lồi khép kín $\mathrm{C}_{\mathrm{i}}$.

Những ưu điểm của kỹ thuật POCS là sự đơn giản và có thể kết hợp với mọi giới hạn cũng như điều kiện cho trước mà phương pháp tiếp cận ngẫu nhiên không cho phép. Tuy nhiên, POCS có mức độ tính toán rất nặng và hội tụ chậm. Giải pháp của POCS cũng không hoàn hảo và phụ thuộc nhiều vào việc đặt thông số ban đầu. Các phương pháp POCS cũng giả định trước các thông số về chuyển động và sự mờ hệ thống. Nó không thể ước tính thông qua những thành phần đã được đăng ký trước và ảnh độ phân giải cao như phương pháp tiếp cận ngẫu nhiên.

\section{3. Đề xuất phương pháp siêu phân giải (SR) trong xử lý ảnh VNREDSAT-1}

Đứng trên phương diện tổng thể thì có hai phương pháp chính là phương pháp dựa trên miền tần số và miền không gian. Phương pháp miền tần số thường sử dụng chuỗi Fourier để mô phỏng dịch chuyển đồng thời đa ảnh ban đầu. Tuy nhiên, do tính chất phức tạp của dịch chuyển đa ảnh nên hàm mô phỏng thường không đủ tham số do đó việc giải bài toán rất khó khăn và mô hình không được chặt chẽ. Do đó phương pháp này ít được phát triển nâng cao. Phương pháp sử dụng miền không gian với đặc điểm là ước lượng các thông số trước dựa trên các thông tin về ảnh và yêu cầu về ảnh độ phân giải cao đầu ra đã giải quyết vấn đề không đủ tham số của phương pháp miền tần số. Đây là hướng đi được phát triển mở rộng mạnh mẽ trong nghiên cứu siêu phân giải. Từ trước đến nay, phương pháp miền không gian có nhiều phương pháp nhưng đó là sự phát triển nối tiếp nhau, phương pháp giải quyết những vấn đề tồn tại của phương pháp trước. Xét về kỹ thuật thì có hai hướng đi chính đó là phương pháp sử dụng biến ngẫu nhiên ước lượng thông qua hàm xác xuất và phương pháp ước lượng các biến thông qua xác định tập lồi bằng phép chiếu tập lồi với các điều kiện biên được xác định trước. Ngoài ra, phương pháp kết hợp cả hai phương pháp trên cũng được phát triển và những kết quả nghiên cứu ban đầu mang lại kết quả tương đối khả quan.

Vì vậy, dựa trên nguyên lý phương pháp sử dụng miền không gian thì để xử lý ảnh VNREDSat-1 cho mục đích nâng cao, đề xuất sử dụng các bước cơ bản sau: 
- Thu nhận đa ảnh trên cùng một (01) vị trí;

- Mô phỏng mối quan hệ giữa các ảnh thu nhận được, thường là thông qua các mô hình chuyển động giữa các ảnh với đối tượng chụp;

- Phân chia Pixel, thường chia một (01) pixel ban đầu thành bốn (04) sub-pixels;

- Lập ma trận các giá trị pixel ban đầu để xác định các giá trị pixel của ảnh độ phân giải cao kết quả;

- Tái cấu trúc ảnh.

Quá trình tính toán thường không phân chia rõ rệt thành các bước như trên mà có thể thực hiện từng bước hoặc thiết lập một mô hình thống nhất kết hợp tất cả các bước trên.

\section{Kết luận}

Những vấn đề trở ngại lớn đối với phương pháp SR xuất phát từ quá trình khớp ảnh, việc tính toán rất nặng của quá trình tính toán tham số mô hình phục hồi ảnh.... Khớp ảnh quyết định chất lượng lấy mẫu không gian bổ sung vào quá trình phân chia pixel và tái cấu trúc. Kỹ thuật khớp ảnh cần phải hết sức lưu tâm với ảnh viễn thám tại những vùng địa hình biến đổi phức tạp. Nhiễu gây ra bởi các sai số trong quá trình khớp ảnh ảnh lớn hơn hiệu ứng mờ do nội suy. Độ chính xác phân chia các subpixel quyết định giá trị pixel ảnh độ phân giải cao. Do đó, để phù hợp với việc tái cấu trúc ảnh SR thì việc khớp ảnh LR có thể giải quyết cùng với trượt xoắn ảnh trước khi tái cấu trúc ảnh để thu được ảnh có độ phân giải không gian cao hơn.

Một hạn chế ứng dụng thực tế của phương pháp tái cấu trúc SR là tính toán đòi hỏi thao tác với các ma trận hết sức tốn bộ nhớ máy tínhTrên thực tế, với ảnh viễn thám thì khối lượng pixel ảnh lớn chính là vấn đề đáng quan tâm. Tuy nhiện, các nhà khoa học cũng đã đề xuất nhiều biện pháp tính toán nhằm giảm bộ nhớ đồng thời tăng tốc độ tính toán lên thông qua việc lược bỏ các thành phần không cần thiết, xây dựng mô hình chặt chẽ từ đầu để loại trừ bớt các thông số đã biết vào tính toán.

Đến nay, để đưa ra được hạn chế cho tất cả các kỹ thuật SR là rất khó. Việc sử dụng phương pháp SR cho ảnh VNREDSat-1 là một nhiệm vụ phức tạp bao gồm nhiều bước tính toán phụ thuộc lẫn nhau.

Bài báo là kết quả của đề tài "Nghiên cứu phương pháp xử lý nâng cao chất lượng ảnh vệ tinh VNREDSat-1", mã số VT/UD-01/13-15, thuộc Chương trình khoa học công nghệ độc lập cấp Nhà nước về Công nghệ vũ trụ mã số KHCN-VT/12-15.O

\section{Tài liệu tham khảo}

[1]. R. Y. Tsai and T. S. Huang. Multipleframe image restoration and registration.In Advances in Computer Vision and Image Processing, pages317-339. Greenwich, CT: JAI Press Inc., 1984.

[2]. M. Elad S. Farsiu, D. Robinson and P. Milanfar. Advances and chal-lenges in superresolution. International Journal of Imaing Systems andTechnology, 14(2):47-57, 2004.

[3]. Sean Borman and Robert L. Stevenson. Super-resolution from image sequences A review. In Proceedings of the 1998 Midwest Symposium on Circuits and Systems, pages 374-378, 1998. 
[4]. S. Baker and T. Kanade. Limits on super-resolution and how to break them. IEEE Transactions on Pattern Analysis and Machine Intelligence, 24(9):1167-1183, 2002.

[5]. M. Elad and A. Feuer. Restoration of single super-resolution image from several blurred, noisy and down-sampled measured images. IEEE Transaction on Image Processing, 6(12):1646-1658, 1997.

[6]. R. C. Hardie, K. J. Barnard, and E. E. Armstrong. Join MAP registration and high resolution image estimation using a sequence of undersampled images. IEEE Transactions on Image Processing, 6(12):1621-1633, 1997.

[7]. H. Stark and P. Oskoui. The method of convex projections and its application to image reconstruction in computerized tomography. Control and Applications, 1989. Proceedings. ICCON '89. IEEE International Conference on. tr 241-244, 1989.

[8]. S. Farsiu, D. Robinson, M. Elad, and P. Milanfar. Fast and robust multi-frame superresolution. IEEE Transaction on Image Processing, 13(10):1327-1344, 2004.

[9]. D. Keren, S. Peleg, and R. Brada. Image sequence enhancement using subpixel displacements. In Proceedings of the IEEE Conference on Computer Vision and Pattern Recognition, pages 742-746, 1988.

[10]. H. Ur and D. Gross. Improved resolution from subpixel shifted pictures. CVGIP: Graphical Models and Image Processing, 54(2):181-186, 1992.

[11]. R. C. Hardie, K. J. Barnard, and E. E. Armstrong. Join MAP registration and high resolution image estimation using a sequence of undersampled images. IEEE Transactions on Image Processing, 6(12):1621-1633, 1997.

[12]. D. C. Youla and H. Webb. Image registration by the method of convexprojections: Part 1-thoery. IEEE Transactions on Medical Imaging,1(2):81-94, 1982.O

\section{Summary}

\section{Improving resolution of remote sensing images by super resolution methods}

Dr. Le Quoc Hung, Eng. Truong Thi Tuyet

\section{Vietnam Remote Sensing Center}

The method for improving remote sensing image spatial resolution is the target of the top research scientists in the field of remote sensing. Up to now, the way to improve image spatial resolution by upgrading and manufacture of new advanced sensors that allow taking high spatial resolution images has enormous costs. In addition, spectral resolution was often limited while enhanced spatial resolution. These reasons prompted scientists to discover the method for improving spatial resolution by transformed algorithms to high resolution images based on received images. This article focuses on assessing some superresolution methods which develop so far. The result will be scientific basis for the purpose of enhancing resolution to better use of VNREDSat-1 data.O

Ngày nhận bài: 10/3/2014. 